\title{
GPPS-CH-2020-0068
}

\section{Direct Numerical Simulation of Real-gas Effects within Turbulent Boundary Layers for Fully-developed Channel Flows}

\author{
Tao Chen, Bijie Yang, Miles Robertson and Ricardo Martinez-Botas \\ Turbo Group, Department of Mechanical Engineering, Imperial College London \\ London SW7 2AX, UK
}

\begin{abstract}
Real-gas effects have a significant impact on compressible turbulent flows of dense gases, especially when flow properties are in proximity of the saturation line and/or the thermodynamic critical point. Understanding of these effects is key for the analysis and improvement of performance for many industrial components, including expanders and heat exchangers in organic Rankine cycle systems.
\end{abstract}

This work analyzes the real-gas effect on the turbulent boundary layer of fully developed channel flow of two organic gases, R1233zd(E) and MDM - two candidate working fluids for ORC systems. Compressible direct numerical simulations (DNS) with real-gas equations of state are used in this research. Three cases are set up for each organic vapour, representing thermodynamic states far from, close to and inside the supercritical region, and these cases refer to weak, normal and strong real-gas effect in each fluid.

The results within this work show that the real-gas effect can significantly influence the profile of averaged thermodynamic properties, relative to an air baseline case. This effect has a reverse impact on the distribution of averaged temperature and density. As the real-gas effect gets stronger, the averaged centre-to-wall temperature ratio decreases but the density drop increases. In a strong real-gas effect case, the dynamic viscosity at the channel center point can be lower than at channel wall. This phenomenon can not be found in a perfect gas flow.

The real-gas effect increases the normal Reynolds stress in the wall-normal direction by $7-20 \%$ and in the spanwise direction by $10-21 \%$, which is caused by its impact on the viscosity profile. It also increases the Reynolds shear stress by $5-8 \%$. The real-gas effect increases the turbulence kinetic energy dissipation in the viscous sublayer and buffer sublayer $\left(y^{*}<30\right)$ but not in the outer layer. The turbulent viscosity hypthesis is checked in these two fluids, and the result shows that the standard two-function RANS model $(k-\varepsilon$ and $k-\omega)$ with a constant $C_{\mu}=0.09$ is still suitable in the outer layer $\left(y^{*}>70\right)$, with an error in $\pm 10 \%$.

\begin{tabular}{|c|c|}
\hline \multicolumn{2}{|c|}{ NOMENCLATURE } \\
\hline$c$ & Sonic velocity $[\mathrm{m} / \mathrm{s}]$ \\
\hline e & Inner heat energy $[\mathrm{J} / \mathrm{kg}]$ \\
\hline$f_{i}$ & Body force $\left[\mathrm{m} / \mathrm{s}^{2}\right]$ \\
\hline$k$ & Turbulent kinetic energy $[\mathrm{J} / \mathrm{kg}]$ \\
\hline$\dot{m}$ & Mass flow rate $[\mathrm{kg} / \mathrm{s}]$ \\
\hline$p$ & Pressure $[\mathrm{Pa}]$ \\
\hline$q_{i}$ & Heat conduction $\left[\mathrm{J} /\left(\mathrm{m}^{2} \mathrm{~s}\right)\right]$ \\
\hline$s$ & Specific entropy $[\mathrm{J} / \mathrm{kg} \cdot \mathrm{K}]$ \\
\hline$t$ & Time $[\mathrm{s}]$ \\
\hline$u$ & Velocity in $\mathrm{x}$ direction $[\mathrm{m} / \mathrm{s}]$ \\
\hline V & Velocity in $\mathrm{x}$ direction $[\mathrm{m} / \mathrm{s}]$ \\
\hline$w$ & Velocity in $\mathrm{x}$ direction $[\mathrm{m} / \mathrm{s}]$ \\
\hline$x_{i}$ & Position [m] \\
\hline$C_{\mu}$ & Turbulent model constant \\
\hline$C_{p}$ & Isobaric specific heat capacity $[\mathrm{J} /(\mathrm{kg} \cdot \mathrm{K})]$ \\
\hline$C_{v}$ & Isochoric specific heat capacity $[\mathrm{J} /(\mathrm{kg} \cdot \mathrm{K})]$ \\
\hline$L$ & Channel length scale $[\mathrm{m}]$ \\
\hline$M$ & Molecular weight $[\mathrm{kg} / \mathrm{kmol}]$ \\
\hline$N$ & Number of nodes [-] \\
\hline$P_{k}$ & Turbulent kinetic energy generation rate [W] \\
\hline$R$ & Specific gas constant $[\mathrm{J} /(\mathrm{kg} \cdot \mathrm{K})]$ \\
\hline$T$ & Temperature $[\mathrm{K}]$ \\
\hline$V$ & Specific volume $\left[\mathrm{m}^{3} / \mathrm{kg}\right]$ \\
\hline$Z$ & Compressibility factor [-] \\
\hline Ec & Eckert number [-] \\
\hline $\mathrm{Ma}$ & Mach number [-] \\
\hline $\operatorname{Re}$ & Reynolds number [-] \\
\hline $\operatorname{Pr}$ & Prandtl number [-] \\
\hline
\end{tabular}

$\begin{array}{cl}\text { Greeks } & \\ \gamma & \text { Specific heat ratio [-] } \\ \varepsilon & \text { Turbulent kinetic energy dissipation rate [W] } \\ \eta & \text { Smallest length scale }[\mathrm{m}] \\ \eta_{k} & \text { Kolmogorov length scale }[\mathrm{m}] \\ \eta_{\theta} & \text { Length scale in temperature field [m] }\end{array}$




$\begin{array}{ll}\lambda & \text { Heat conductivity }[\mathrm{W} /(\mathrm{m} \cdot \mathrm{K})] \\ \mu & \text { Dynamic viscosity }[\mathrm{Pa} \cdot \mathrm{s}] \\ v & \text { Kinematic viscosity }\left[\mathrm{m}^{2} / \mathrm{s}\right] \\ \rho & \text { Density }\left[\mathrm{kg} / \mathrm{m}^{3}\right] \\ \tau & \text { Viscous stress }[\mathrm{Pa}] \\ \Gamma & \text { Fundamental derivative }[-]\end{array}$

\section{Subscripts}

$\begin{array}{lll}0 & & \text { Stagnation conditions } \\ c r & & \text { Critical condition } \\ p g & & \text { Perfect gas } \\ t & & \text { Turbulent parameter } \\ W & & \text { Channel wall } \\ B & & \text { Bulk paramer } \\ C & & \text { Channel central layer } \\ G & & \text { Global parameter } \\ \tau & & \text { Viscous parameter }\end{array}$

\begin{tabular}{cll} 
Accents & & \\
\cline { 1 - 1 } & & Non-dimensional parameter \\
$\bar{x}$ & & Reynolds-averaged mean parameter \\
$x^{\prime}$ & & Reynolds-averaged fluctuation \\
$\tilde{x}$ & Favre-averaged mean parameter \\
$x^{\prime \prime}$ & Favre-averaged fluctuation
\end{tabular}

\section{INTRODUCTION}

Wall-bounded turbulent flows of organic fluids have drawn increasing attention in recent years, in part due to the application of organic Rankine cycle (ORC) systems for waste heat recovery. With wall-bounded flows present in the majority of ORC system components, an improved understanding of the detailed fluid mechanics within real-gas boundary layers is fundamental to (a) the validation of Computational Fluid Dynamics (CFD) codes, and therefore (b) the maximisation of component-level performance.

The majority of research within this area [1] [2] [3] [4] has analysed blade-bounded flows in a turbine or nozzle, based upon the Reynolds Averaged Navier Stokes (RANS) simulation method. However further work is required for validation, to confirm that real-gas effects do not disturb the fundamental assumptions contained within RANS simulations.

Real-gas effects distinguish organic fluids from perfect gases such as air. The perfect-gas Equation of State (EoS), $p V=R T$, is inapplicable in these fluids when they work close to the saturation curve and/or critical point. In this case, the thermodynamic properties such as pressure, viscosity, and heat capacity, will depend not only on temperature, but also on density. As a result of this, many perfect-gas-based simplifications must be checked before their application. Meanwhile, most frequently-used organic fluids, including refrigerants, siloxanes and alkanes, have a large molecular weight and a high-level molecular complexity, also called dense gases [5] [6].

Work by Colonna \& Guardone [7] has shown that as the gas molecular complexity increases, its heat capacity, $C_{v} / R$, will increase, and the fundamental derivative of gas dynamics, $\Gamma$ (defined in Eq. 1 [8]) will decrease. Perfect gases always have a constant $\Gamma=(\gamma+1) / 2$ of more than unity, while dense gas can have a variable and lower $\Gamma$. For most of the frequently-used organic fluids, the minimum $\Gamma$ can be lower than 1 , where the speed of sound will decrease with pressure in an isentropic process [8]. There are also a group of theoretical BZT fluids (named after Bethe [9], Zel'dovich [10] and Thompson [8]), which contain a region of negative $\Gamma$. In that region an 'inversion' of typical supersonic behaviour is observed, with flows achieving compression via fans, as opposed to shock waves [8]. To summarise, real-gas and dense-gas effects can have a significant influence on the mechanics of an organic fluid - it is the purpose of this work to study these effects in detail.

$$
\Gamma \equiv \frac{c^{4}}{2 V^{3}}\left(\frac{\partial^{2} V}{\partial p^{2}}\right)_{s}
$$

This work studies the real-gas effect on the turbulent boundary layer in a fully developed channel flow by means of Direct Numerical Simulation (DNS), in which no turbulence model is required due to flows being resolved down to the Kolmogorov microscale. DNS helps to get rid of the uncertainty caused by any turbulent modelling assumption, and produces the realization of a real-gas flow.

Kim, Moin \& Moser [11] firstly applied the DNS method on a incompressible fully developed channel flow at Reynolds number of 3300. They studied the turbulence statistics near the wall, and found that their results showed a good agreement with the experimental data.

Huang, Coleman \& Bradshaw [12] studied compressible flow within a channel, and analysed the compressibility-associated terms in Reynolds averaged energy equations. They found that the averaged property profiles matched the corresponding incompressible curves well, by scaling by mean density, $\bar{\rho} / \rho_{w}$. They also proposed the model for Reynolds analogy, mean-Favre-averaged fluctuations, and pressure-dilatation terms.

Patel et al [13] studied a turbulent channel with variable properties, in different cases of the relation between dynamic viscosity and temperature. They found that the normal Reynolds stress anisotropy and turbulence-to-mean time scales were influenced by that relation.

Sciacovelli, Cinnella, \& Gloerfelt [14] studied a dense gas, $\mathrm{PP}_{11}\left(\mathrm{C}_{14} \mathrm{~F}_{24}\right)$, in a turbulent channel flow. They focused on one operating point inside the region $\Gamma<1$, and found the mean temperature is nearly constant and the mean viscosity decreases from the wall to the centreline, showing a liquid-like behavior. Their results showed that dense gas channel flows can tend towards the behaviour of an incompressible channel flow with variable properties, due to their heat capacity.

The study within this work focuses on two commonly-used organic fluids, and compares three different working conditions of each fluid to give insight into the impact of real-gas effects. The main properties of these two organic fluids are show in Tab. 1. Refrigerant R1233zd(E), trans-1-chloro-3,3,3-trifluoropropene $\left(\mathrm{CF}_{3} \mathrm{CH}=\mathrm{CHCl}\right), \quad$ is accepted as a high-efficiency and 
environmental-friendly fluid for ORC systems, and it can be a substitute of refrigerant R245fa. Meanwhile, MDM, formally known as octamethyltrisiloxane $\left(\mathrm{C}_{8} \mathrm{H}_{24} \mathrm{O}_{2} \mathrm{Si}_{3}\right)$, is a representative of the group of siloxane fluids, since they also can be a potential fluid of ORC systems.

Table 1 KEY PROPERTIES OF R1233zd(E) AND MDM [15].

\begin{tabular}{cll} 
Property & R1233zd(E) & MDM \\
\hline$T_{c r}(\mathrm{~K})$ & 439.6 & 564.1 \\
$p_{c r}(\mathrm{kPa})$ & 3624 & 1415 \\
$\rho_{c r}\left(\mathrm{~kg} / \mathrm{m}^{3}\right)$ & 480.2 & 256.7 \\
$M(\mathrm{~kg} / \mathrm{kmol})$ & 130.5 & 236.5
\end{tabular}

\section{METHODOLOGY}

\section{Numerical Method}

A fully developed channel flow is simulated in the domain shown in Fig. 1, where the boundaries on $\mathrm{X}$ and $\mathrm{Z}$ direction are periodic boundaries and the boundary on $\mathrm{Y}$ direction is no-slip isothermal wall boundary - mean flow is in the $\mathrm{X}$ direction. The scale of the domain is $L_{x} \times L_{y} \times L_{z}=2 \pi h \times 2 h \times \pi h$, where $\mathrm{h}$ is the characteristic length of the channel.

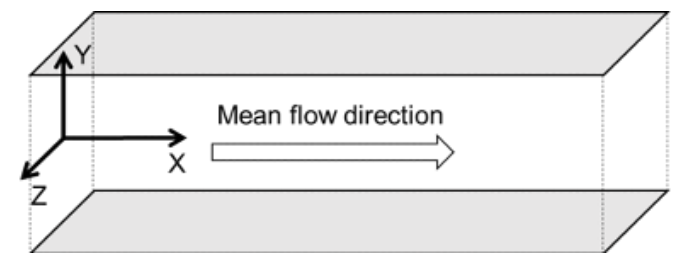

Fig.1 CALCULATION DOMAIN OF THE CHANNEL FLOW SIMULATION.

This work is based on the solution of the three-dimensional Navier-Stokes (NS) equations (Eq.2-4), which are closed by the equation of state $p=p(\rho, T)$. These equations are non-dimensionalised by half-channel height, $h$, globally averaged density, $\rho_{G} \equiv \frac{1}{h} \int_{0}^{h} \bar{\rho} d y$, and global averaged velocity, $u_{G} \equiv$ $\frac{1}{h \rho_{G}} \int_{0}^{h} \overline{\rho u} d y$, before being calculated.

$$
\begin{gathered}
\frac{\partial \rho}{\partial t}+\frac{\partial \rho u_{j}}{\partial x_{j}}=0 \\
\frac{\partial \rho u_{i}}{\partial t}+\frac{\partial \rho u_{j} u_{i}}{\partial x_{j}}=-\frac{\partial p}{\partial x_{i}}+\frac{\partial \tau_{i, j}}{\partial x_{j}}+\rho f_{i} \\
\frac{\partial \rho\left(e+\frac{1}{2} u_{i}^{2}\right)}{\partial t}+\frac{\partial \rho u_{j}\left(e+\frac{1}{2} u_{i}^{2}\right)}{\partial x_{j}} \\
=-\frac{\partial u_{j} p}{\partial x_{j}}+\frac{\partial u_{i} \tau_{i, j}}{\partial x_{j}}-\frac{\partial q_{j}}{\partial x_{j}}+\rho u_{j} f_{j}
\end{gathered}
$$

where:

$$
\tau_{i j}=\mu\left(\frac{\partial u_{i}}{\partial x_{j}}+\frac{\partial u_{j}}{\partial x_{i}}\right)-\frac{2}{3} \mu \nabla \cdot u_{k} \delta_{i j}
$$

$$
q_{i}=-\lambda \frac{\partial T}{\partial x_{i}}
$$

Non-dimensional parameters can be declared as:

$$
\begin{aligned}
\hat{t} & \equiv t \frac{u_{G}}{h} \\
\hat{x}_{i} & \equiv \frac{x_{i}}{h} \\
\hat{u}_{i} & \equiv \frac{u_{i}}{u_{G}} \\
\hat{\rho} & \equiv \frac{\rho}{\rho_{G}} \\
\hat{T} & \equiv \frac{T}{T_{w}} \\
\hat{p} & \equiv \frac{p}{\rho_{G} u_{G}^{2}} \\
\hat{e} & \equiv \frac{e}{u_{G}^{2}} \\
\hat{\mu} & \equiv \frac{\mu}{R e_{G} \mu_{G}} \\
\hat{\lambda} \equiv \frac{T_{w} \lambda}{\rho_{G} u_{G}^{3} h} & =\frac{C_{p, G} T_{w} \lambda}{R e_{G} P r_{G} u_{G}^{2} \lambda_{G}}
\end{aligned}
$$

For a real gas, all the thermodynamic parameters are two-variable functions of both temperature and density. The global density and wall temperature $\left(\rho_{G}, T_{w}\right)$ are taken to determinate the global dynamic viscosity, $\mu_{G}$, and heat capacity $C_{p, G}$ during the non-dimensionalisation. The global Reynolds number is defined by $R e_{G} \equiv \frac{\rho_{G} u_{G} h}{\mu_{G}}$ and the global Prandtl number is defined by $\operatorname{Pr}_{G} \equiv \frac{C_{p, G} \mu_{G}}{\lambda_{G}}$. Subsequently, expression of the non-dimensional NS equations is the same as the original expression.

For the fully developed channel flow there is a periodic boundary instead of inlet and outlet boundaries, so the pressure drop along the stream is balanced by an artificial added body force $f_{i}$, which is equivalent to the mean pressure gradient in physical flow [12]. The body force $f_{i}$ is non-zero only for the mean flow direction $(i=1)$, and it is uniform in the domain. The body force is calculated in each time step by keeping the global averaged mass flow rate invariant in the calculation.

The DNS solver is developed based on a compressible DNS code from X. Li [16] with an addition of a real-gas model. All the thermal properties in this model are two-variable functions of the local density and temperature, and they are calculated by the state-of-art Helmholtz energy EoS in REFPROP [15]. Organic fluid cases are calculated by this real-gas model while the air case is calculated by a perfect-gas model. The NS equations are solved after non-dimensionalisation. For spectral discretisation, the convective flux derivatives are calculated by seventh-order upwind 
finite-difference scheme, and the viscous flux derivatives are calculated by sixth-order central scheme. For time advancement, it is solved by a third-order Runge-Kutta method. The validation of the simulation method is shown in the appendix.

The number of nodes in this work is $N_{x} \times N_{y} \times N_{z}=128 \times 257 \times$ 128 , and the overall number of nodes is $4.2 \times 10^{6}$. The spatial resolution is chosen as DNS requirements. Zonta [17] advised that $(\Delta x / \eta)_{\text {max }} \approx 12,(\Delta y / \eta)_{\text {max }} \approx 2$ and $(\Delta z / \eta)_{\text {max }} \approx 6$, while Lee [18] advised that $(\Delta x / \eta)_{\max } \approx 12.4,(\Delta y / \eta)_{\max } \approx 3.0$ and $(\Delta z / \eta)_{\max } \approx 7.9$, where $\eta$ is the smallest length scale in the flow. $\eta$ is equal to the Kolmogorov length scale $\eta_{k}$ when Prandtl number is lower than 1 , while it equal to $\eta_{\theta} \approx \eta_{k} / \sqrt{P r}$ when Prandtl number is higher than 1 [19]. The spatial resolution of cases in this work is shown in Tab. 2, and it can fulfill the DNS requirement. The $y^{+}$ of the first layer from wall boundary is below 0.5 , and the first 10 layers are within $y^{+}=5$.

Figure 2 (a) and (b) show two-point correlation of velocity $\mathrm{u}$ and density $\rho$ between points in distance $\Delta x \in\left[0, L_{x} / 2\right]$ at $y^{+} \approx 10$, and Fig. 2 (c) and (d) show the two-point correlation between points in distance $\Delta z \in\left[0, L_{z} / 2\right]$. It shows that all the two-point correlations are close to zero at $L_{x} / 2$ and $L_{z} / 2$, which means that the domain size $L_{x}$ and $L_{z}$ are large enough for the channel flow cases with periodic boundary.

This work is solved by the High Performance Computer in Imperial College London. Each case uses 512 CPUs, and it takes 10-20 days to get converged. After that, the statistic is taken every $\Delta \hat{t}=2$ within a time range $\Delta \hat{t}=1000$.

Table 2 DNS CASES SPATIAL RESOLUTION.

\begin{tabular}{c|l|lll|lll} 
Case & Air & R1 & R2 & R3 & M1 & M2 & M3 \\
\hline$(\Delta x / \eta)_{\max }$ & 12.6 & 9.9 & 11.1 & 14.3 & 9.4 & 9.4 & 10.6 \\
$(\Delta y / \eta)_{\max }$ & 0.8 & 1.0 & 1.1 & 1.9 & 1.1 & 1.1 & 1.3 \\
$(\Delta z / \eta)_{\max }$ & 6.3 & 5.0 & 5.6 & 7.1 & 4.7 & 4.7 & 5.3
\end{tabular}

\section{Case Set-Up}

There are no standard inlet and outlet boundaries in this domain for the periodic boundary condition, so the global averaged density $\rho_{G}$ and mass flow rate $\dot{m}_{G}=u_{G} h \rho_{G}$ are set as an alternative. The global averaged velocity is given as $u_{G}=M a_{G} c_{G}$, where $M a_{G}$ is the global Mach number. $c_{G}$ is a reference global sound speed defined by wall temperature and global averaged density $c_{G}=c_{r g}\left(\rho_{G}, T_{w}\right)$ for organic fluids, and only wall temperature $c_{G}=c_{p g}\left(T_{w}\right)$ for air. The dynamic viscosity $\mu_{G}$ is defined in the same fashion $\mu_{G}=\mu_{r g}\left(\rho_{G}, T_{w}\right)$ for organic fluids and $\mu_{G}=\mu_{p g}\left(T_{w}\right)$ for air. $\mu_{r g}$ is calculated by the real-gas model, and $\mu_{p g}$ is calculated by the Sutherland viscosity law [20] shown in Eq. 16, where $T_{s}=110.56$ $\mathrm{K}$ is the effective temperature.

$$
\frac{\mu_{p g}}{\mu_{w}}=\left(\frac{T}{T_{w}}\right)^{3 / 2} \frac{T_{w}+T_{S}}{T+T_{S}}
$$

The initial condition is a turbulent flow field, in which the global averaged density $\rho_{G}$ and mass flow rate $\dot{m}_{G}$ are set. During the calculating, $\rho_{G}$ and $\dot{m}_{G}$ will not change. After each time step, $\rho_{G}$ is calculated and compared with its value in the previous step. If there is a gap between them (caused by numerical error), the density at all points will plus or minus a same small density source term $\Delta \rho$ so as to keep $\rho_{G}$ invariant after this step. $\dot{m}_{G}$ is also calculated in each step and compared with its value in the previous step. If they are different, the body force will be changed so as to maintain an invariant $\dot{m}_{G}$.

The global Mach number $M a_{G} \equiv \frac{u_{G}}{c_{G}}$ and global Reynolds number $\operatorname{Re}_{G} \equiv \frac{\rho_{G} u_{G} h}{\mu_{G}}$ are set at 2.25 and 4880 for all seven cases. The air case follows the perfect-gas Equation of State, requiring only one thermodynamic condition - the wall temperature, $T_{w}=$ $288 \mathrm{~K}$. The two thermodynamic conditions chosen for the real-gas cases were wall temperature and global density. From R1 to R3, the set-up point moves closer to the supercritical region, shown by the temperature-entropy diagram in Fig. 3. The red and blue lines in Fig. 3 are the averaged thermodynamic property distribution in each real-gas case. $C_{v} / R$ for each case is list in Tab. 3, and the value for MDM is the largest whilst air is the smallest. As the set-up point closer to the supercritical region $C_{v} / R$ increases, although this change is small in comparison to the difference in values between R1233zd(E) and MDM. The fundamental derivative $\Gamma$ shows that the minimum value is at $\mathrm{R} 2$ and $\mathrm{M} 2$ for the two organic fluids, respectively.

Table 3 CALCULATING CONDITIONS FOR CASE SET-UP (R = R1233zd(E), $\mathrm{M}=\mathrm{MDM})$.

\begin{tabular}{c|l|lll|lll} 
Case & Air & $\mathbf{R 1}$ & $\mathbf{R 2}$ & $\mathbf{R 3}$ & M1 & M2 & M3 \\
\hline$T_{w} / T_{c r}$ & - & 0.86 & 0.98 & 1.03 & 0.86 & 0.98 & 1.03 \\
$\rho_{G} / \rho_{c r}$ & - & 0.02 & 0.27 & 1.00 & 0.02 & 0.27 & 1.00 \\
$R e_{G}$ & 4880 & 4880 & 4880 & 4880 & 4880 & 4880 & 4880 \\
$M a_{G}$ & 2.25 & 2.25 & 2.25 & 2.25 & 2.25 & 2.25 & 2.25 \\
$C_{v, G} / R$ & 2.5 & 13.4 & 15.7 & 17.3 & 53.1 & 58.3 & 61.9 \\
$\Gamma$ & 1.2 & 1.01 & 0.82 & 1.90 & 0.97 & 0.59 & 1.62 \\
$Z$ & 1 & 0.96 & 0.67 & 0.32 & 0.96 & 0.68 & 0.33
\end{tabular}

\section{RESULTS AND ANALYSIS}

\section{Averaged Velocity and Thermodynamic Properties}

The variables are averaged on each $\mathrm{y}$ level, since the $\mathrm{x}$ and $\mathrm{z}$ directions are periodic. For analysis, the wall shear stress is defined as the averaged value $\tau_{w} \equiv \overline{[\mu(\partial u / \partial y)}_{w}$ and wall friction velocity is defined as $u_{\tau} \equiv \sqrt{\tau_{w} / \bar{\rho}_{w}}$. Huang et al [12] proposed a semi-local friction velocity $u_{\tau}^{*} \equiv \sqrt{\tau_{w} / \bar{\rho}}$ as well, which takes compressibility effects into consideration.

Some important global results of each case are listed in Tab. 4. Bulk Reynolds number and bulk Mach number are defined as $R e_{B} \equiv$ $\rho_{G} u_{G} h / \bar{\mu}_{w}$ and $M a_{B} \equiv u_{G} / \bar{c}_{w}[14]$, and they are different from $R e_{G}$ and $M a_{G}$ in real gases $\left(\bar{\mu}_{w} \neq \mu_{G}\right)$.

The centre-line Reynolds number and Mach number are defined as $\operatorname{Re}_{C} \equiv \bar{\rho}_{C} \bar{u}_{C} h / \bar{\mu}_{C}$ and $M a_{C} \equiv \overline{u_{C} / c_{C}}$. The values show that $\operatorname{Re}_{B}$ decreases as the set-up point is closer to the supercritical region (from R1 to R3 and from M1 to M3), while $R e_{C}$ increases. Both organic fluids have smaller $R e_{B}$ and larger $R e_{C}$ relative to the 


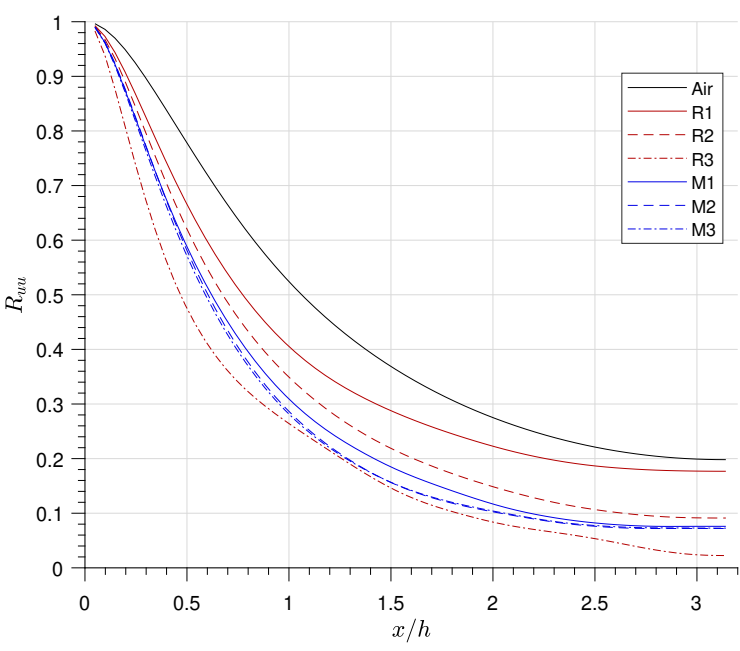

(a)

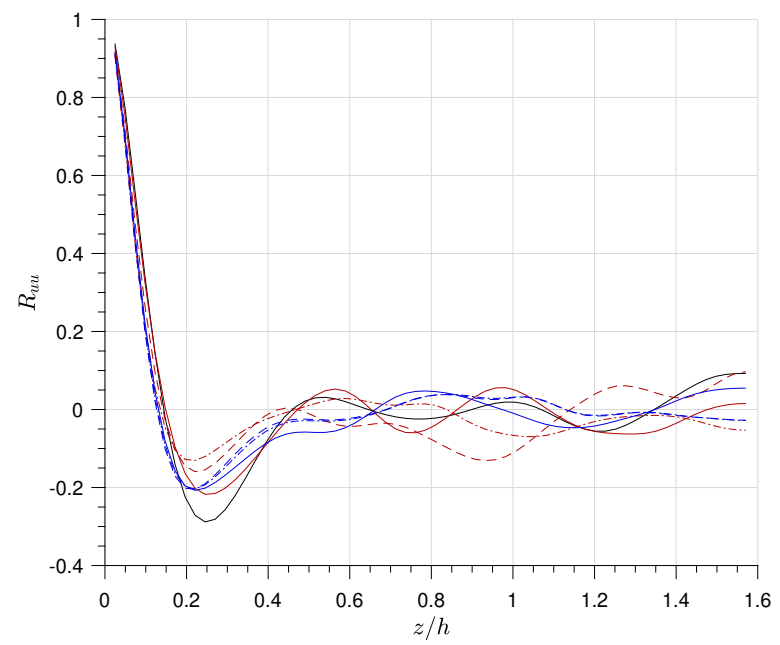

(c)

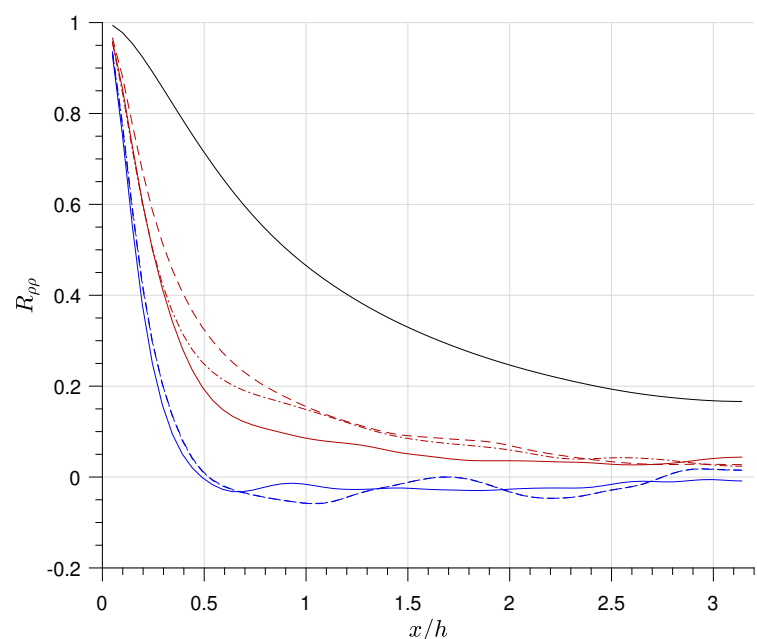

(b)

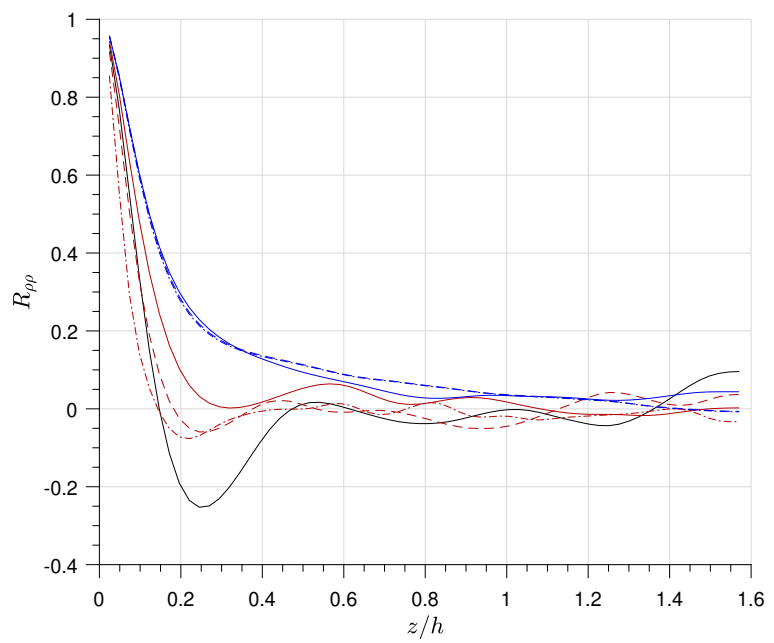

(d)

Fig.2 TWO-POINT CORRELATION OF VELOCITY U AND DENSITY IN STREAMWISE AND SPANWISE. CORRELATION IN STREAMWISE (a),(b); CORRELATION IN SPANWISE (c),(d).

air baseline. The Mach number $M a_{B}$ firstly increases and then decreases as the set-up point is closer to the supercritical region (from R1 to R3 and from M1 to M3), while the centreline Mach number shows minimal change across cases.

The friction Reynolds number is defined as $\operatorname{Re}_{\tau} \equiv \bar{\rho}_{w} u_{\tau} h / \bar{\mu}_{w}$. The friction Reynolds number is lower in the organic fluid cases than air, while lowest in the supercritical cases (R3 and M3). The semi-local friction Reynold number $R e_{\tau}^{*} \equiv \bar{\rho} u_{\tau}^{*} h / \bar{\mu}$ has a higher value for the two organic gases than air, and $R e_{\tau}^{*}$ is highest in the supercritical cases R3 and M3 for each fluid.

Reynolds averaged velocity and distribution versus non-dimensional wall distance $y^{+} \equiv \bar{\rho}_{w} u_{\tau} y / \bar{\mu}_{w}$ is shown in Fig. 4(a) The position $y^{+}=0$ is the wall, while the maximum $y^{+}$is the central layer of the channel. It shows that the organic fluid cases are closer to the $\log$ law line $u^{+}=0.25 \ln \left(y^{+}\right)+5.5$ in the outer layer than air. In each fluid, as the case moves closer to the supercritical region, the velocity distribution increases in deviation from the log law line. After the application of a Van
Driest transformation (Eq. 17) [21] good agreement to the log law in the outer layer is observed (shown in Fig 4(b)).

$$
\bar{u}_{V D}=\int_{0}^{u} \sqrt{\frac{\bar{\rho}}{\bar{\rho}_{w}}} d u
$$

The averaged temperature, density and pressure along the wall-normal direction are shown in Fig. 5, where the abscissa is the non-dimensional wall distance $y^{*} \equiv \bar{\rho} u_{\tau}^{*} y / \bar{\mu}$. Figure 5(a) shows that the temperature in the central region of the channel is higher than wall temperature, and centre-to-wall temperature ratio $\bar{T}_{C} / T_{w}$ is lower in the organic fluid cases than in the air case (shown in Tab. 4).

For each organic fluid, $\bar{T}_{C} / T_{w}$ decreases as the point moves closer to the supercritical region (R1 to R3 and M1 to M3). $\bar{T}_{C} / T_{w}$ correlated positively to $\operatorname{PrEc}$ in these cases (Prandtl number $\mathrm{Pr} \equiv$ $C_{p} \mu / \lambda$, Eckert number $\left.E c \equiv c^{2} / C_{p} T\right)$, which means the larger $\operatorname{PrEc}$ the larger $\bar{T}_{c} / T_{w}$ will be. 


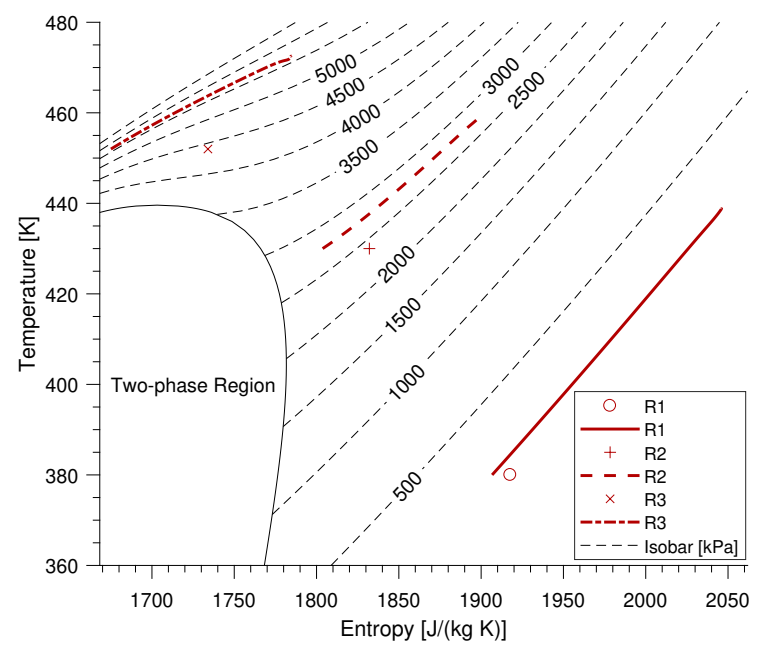

(a) R1233zd(E)

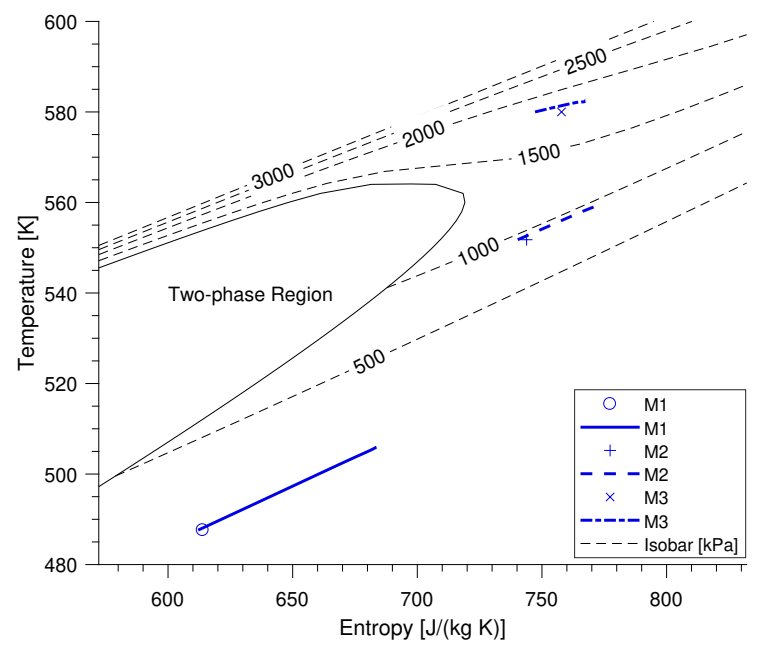

(b) MDM

Fig.3 TEMPERATURE-ENTROPY DIAGRAM OF R1233zd(E) AND MDM. DOTS REFER TO SET-UP CASES, WHILST LINES REFER TO MEAN THERMAL PROPERTY DISTRIBUTION.

When averaging and integrating the energy equation, the heat transfer as a function of wall distance can be represented by Eq. 18 [12]. In the expression, $F_{u}(y)$ is a velocity distribution function which equal to 0 at $\hat{y}=0$ and equal to 1 at $\hat{y}=1$. Since the heat conductivity fluctuation is small compared with averaged conductivity, the gradient of averaged temperature can be derived to Eq. 19.

By assuming the velocity distribution function and the thermal conductivity ratio the same in all these cases, the non-dimensional temperature gradient is approximately proportional to the global Prandtl number multiplied by global Eckert number $\operatorname{Pr}_{G} E c_{G}$ whose values are shown in Tab. 4. For a perfect gas, $E c_{G}=\gamma R / C_{p}=\gamma-1$. The organic fluids have higher molecular complexity, leading to a larger $C_{p} / R$ and therefore a smaller $E c$. For each organic fluid, $P r$ and $E c$ are variable, and the closer to the super critical region the smaller the value of $\operatorname{PrEc}$.

$$
\overline{\hat{\lambda} \frac{\partial \hat{T}}{\partial \hat{y}}}=\hat{\tau}_{w} \int_{\hat{y}}^{1} \bar{\rho} \hat{u} d \hat{y} \frac{\bar{q}}{\bar{q}+\bar{q}_{t}}=\hat{\tau}_{w} F_{u}(\hat{y}) \frac{\bar{q}}{\bar{q}+\bar{q}_{t}}
$$

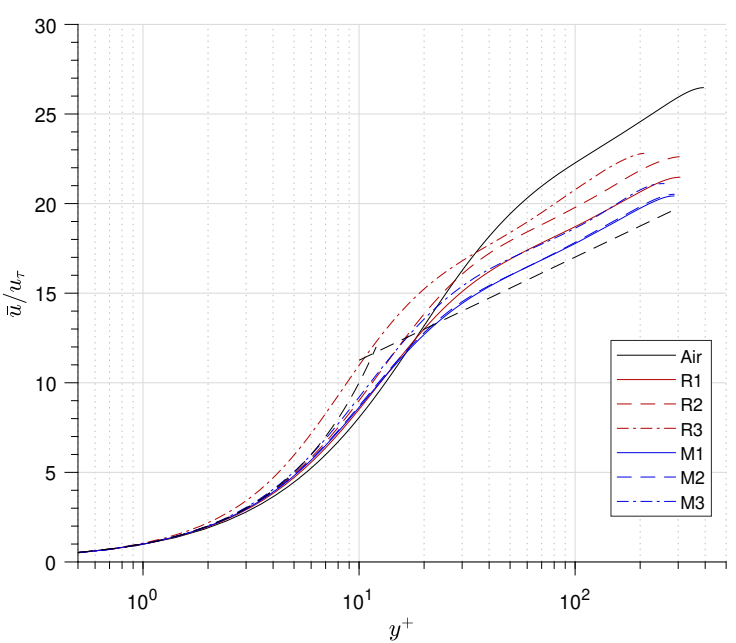

(a)

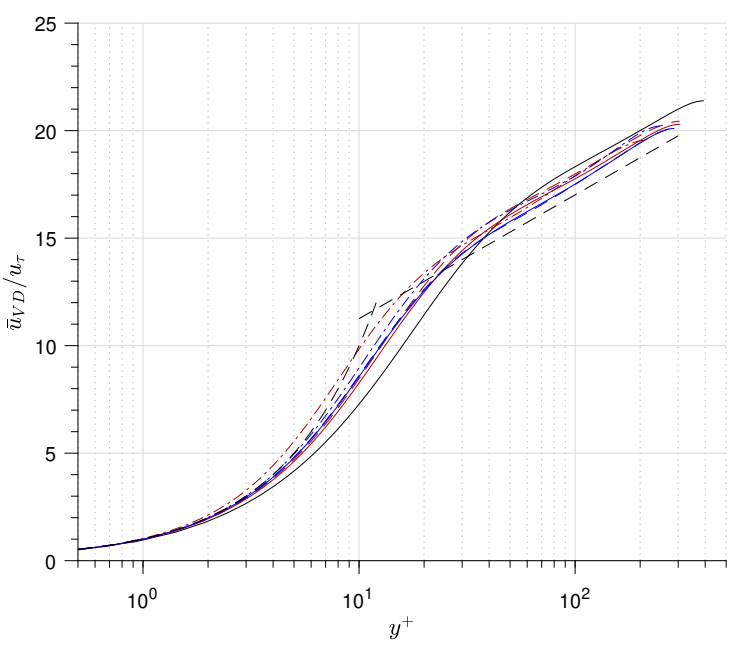

(b)

Fig.4 DISTRIBUTION OF X-DIRECTION AVERAGED VELOCITY ALONG WALL DISTANCE. BLACK DASHED LINES REFER TO $u^{+}=y^{+}$IN THE VISCOUS SUBLAYER AND $u^{+}=0.25 \ln \left(y^{+}\right)+5.5$ IN THE OUTER LAYER.

$$
\begin{gathered}
\frac{\overline{\partial \hat{T}}}{\partial \hat{y}} \approx \frac{\hat{\tau}_{w} F_{u}(\hat{y})}{\hat{\lambda}} \frac{\bar{q}}{\bar{q}+\bar{q}_{t}} \\
\frac{\overline{\partial \hat{T}}}{\partial \hat{y}} \propto \frac{1}{\hat{\lambda}}=\frac{\operatorname{Re}_{G} \operatorname{Pr}_{G} u_{G}^{2} \lambda_{G}}{C_{p, G} T_{w} \lambda} \propto \operatorname{Pr}_{G} E c_{G}
\end{gathered}
$$

The density is higher at the channel wall than the channel centre (shown in Fig. 5(c), and the value of the wall-to-centre density ratio $\bar{\rho}_{w} / \bar{\rho}_{C}$ is shown in Tab. 4. The largest density ratio also appears in the air case, which is the same for the temperature ratio mentioned above. However, for each organic fluid, the density ratio increases as the set-up point moves closer to the supercritical region, while the temperature ratio has a reverse trend.

Since the pressure is nearly constant $\left(\bar{p} / \bar{p}_{w}>0.985\right.$ shown in Fig. 5(d)), the density gradient is approximately equal to the temperature gradient multiplied by $-\left(\frac{\partial \hat{\rho}}{\partial \hat{T}}\right)_{p}$ (Eq. 21). The value 


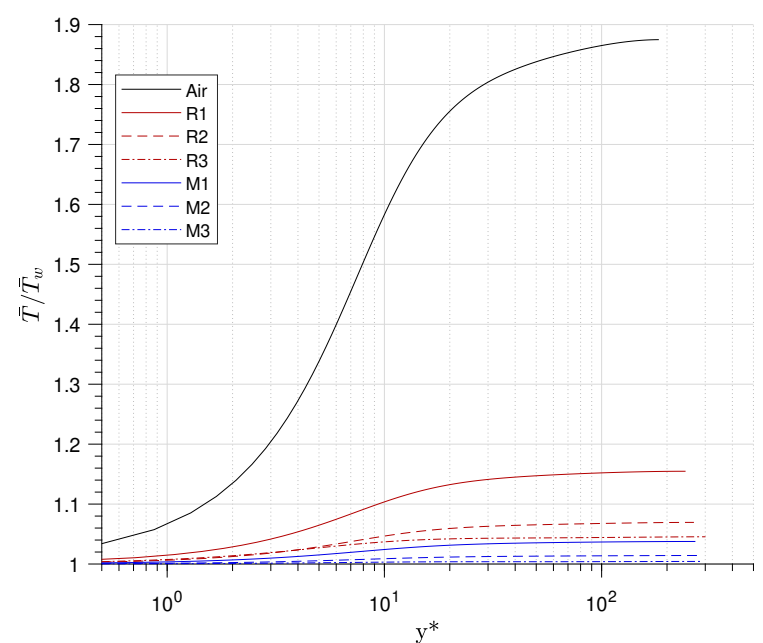

(a) Averaged temperature

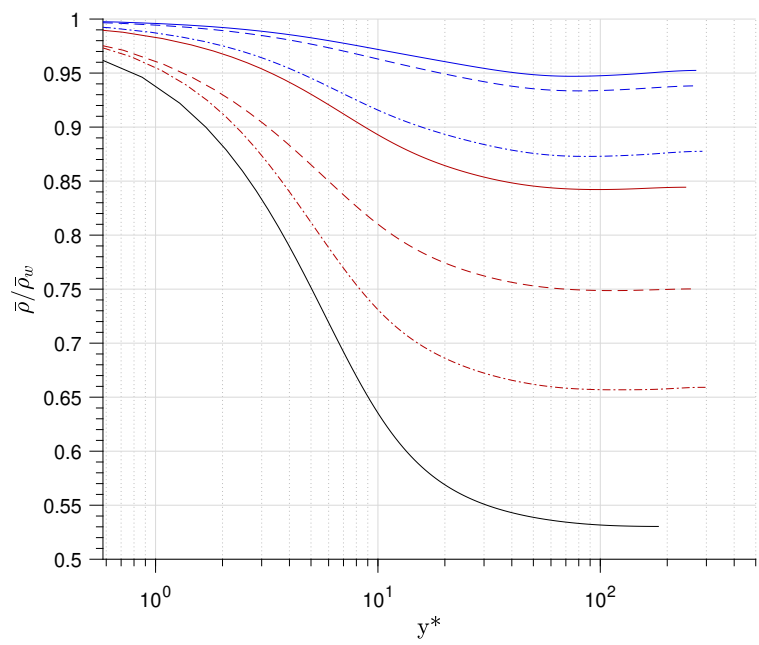

(c) Averaged density

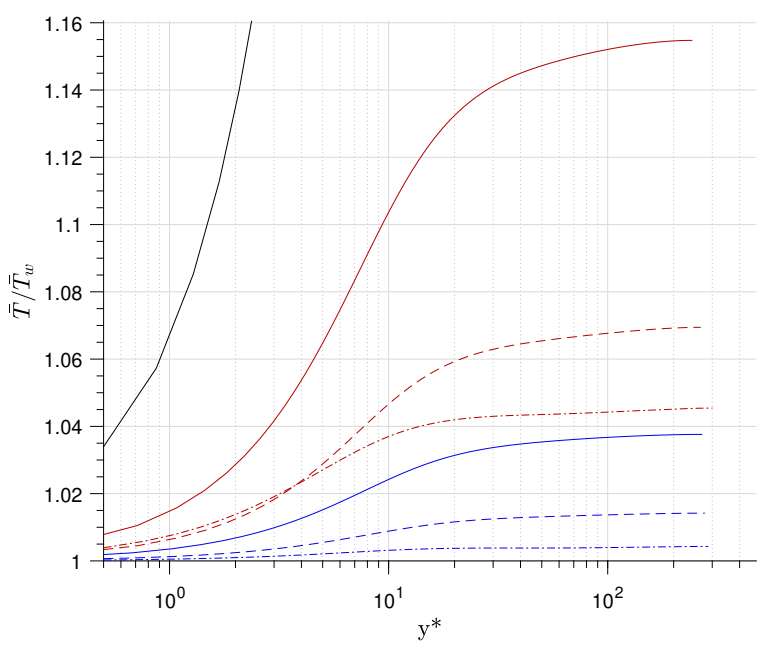

(b) Averaged temperature (zoomed)

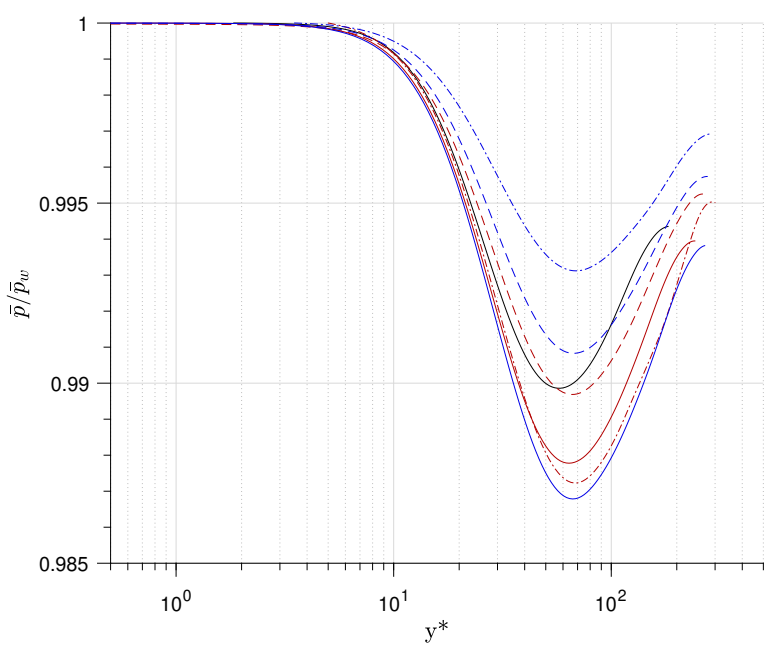

(d) Averaged pressure

Fig.5 DISTRIBUTION OF AVERAGED TEMPERATURE, DENSITY AND PRESSURE ALONG THE WALL-NORMAL DIRECTION.

Table 4 DNS RESULTS FOR EACH CASE

\begin{tabular}{c|l|lll|lll} 
Case & Air & $\mathbf{R 1}$ & $\mathbf{R 2}$ & $\mathbf{R 3}$ & $\mathbf{M 1}$ & $\mathbf{M 2}$ & $\mathbf{M 3}$ \\
\hline$R e_{B}$ & 4880.0 & 4881.1 & 4530.4 & 2683.1 & 4880.7 & 4836.4 & 4175.0 \\
$R e_{C}$ & 3512.5 & 4824.6 & 5219.2 & 5648.0 & 5394.9 & 5519.9 & 5632.4 \\
$M a_{B}$ & 2.25 & 2.26 & 2.52 & 1.26 & 2.25 & 2.32 & 2.09 \\
$M a_{C}$ & 1.92 & 2.40 & 2.34 & 2.21 & 2.53 & 2.50 & 2.51 \\
$R e_{\tau, C}$ & 395.9 & 306.9 & 306.2 & 205.7 & 287.6 & 287.9 & 262.9 \\
$R e_{\tau, C}^{*}$ & 183.4 & 243.4 & 266.0 & 300.7 & 270.1 & 277.4 & 287.8 \\
$\bar{T}_{C} / T_{w}$ & 1.875 & 1.155 & 1.069 & 1.045 & 1.038 & 1.014 & 1.004 \\
$\operatorname{Pr}_{G} E c_{G}$ & 0.28 & 0.055 & 0.025 & 0.018 & 0.013 & 0.005 & 0.002 \\
$\operatorname{Pr}_{G}$ & 0.7 & 0.81 & 1.02 & 4.77 & 0.73 & 0.67 & 1.41 \\
$E c_{G}$ & 0.4 & 0.069 & 0.025 & 0.004 & 0.017 & 0.007 & 0.001 \\
$C_{p, G} / R$ & 3.5 & 14.7 & 21.7 & 81.3 & 54.3 & 64.5 & 139.4 \\
$\bar{\rho}_{w} / \bar{\rho}_{c}$ & 1.89 & 1.18 & 1.33 & 1.52 & 1.05 & 1.07 & 1.14 \\
$-\left(\frac{\partial \hat{\rho}}{\partial \hat{T}_{1}}\right)_{p, G}$ & 1 & 1.1 & 3.9 & 31.4 & 1.1 & 3.9 & 33.3 \\
$\hat{p}_{w}$ & 0.26 & 0.22 & 0.28 & 0.27 & 0.21 & 0.31 & 0.43 \\
$\bar{\mu}_{c} / \bar{\mu}_{w}$ & 1.57 & 1.16 & 1.00 & 0.56 & 1.04 & 1.01 & 0.86
\end{tabular}

of $-\left(\frac{\partial \hat{\rho}}{\partial \hat{T}}\right)_{p}$ is shown in Tab. 4. It is equal to 1 in perfect gas, but it increases significantly as the set-up point moves closer to the supercritical region in real gases(from R1 to R3, and M1 to M3). The effect of the increase in $-\left(\frac{\partial \hat{\rho}}{\partial \hat{T}}\right)_{p}$ is larger than that of the decrease in temperature gradient, so the density drop is larger in cases closer to the supercritical region.

$$
-\frac{\overline{d \hat{\rho}}}{d \hat{y}} \approx-\left(\frac{\partial \hat{\rho}}{\partial \hat{T}}\right)_{p} \frac{\overline{d \hat{T}}}{d \hat{y}}
$$

The pressure ratio has a minimum value at $y^{*} \approx 70$ for all the cases (shown in Fig. 5 (d)). As the set-up point moves closer to the supercritical region, the maximum pressure drop firstly increases and then decreases in R1233zd(E) (from R1 to R3), while it increases monotonically in MDM (from M1 to M3). This trend of maximum pressure drop is nearly negatively related to the averaged pressure at wall $\overline{\hat{p}}_{w}$ (shown in Tab. 3), and this relation can be explained by the Reynolds averaged momentum equation in the $y$ direction (Eq. 22) in the following paragraph. 


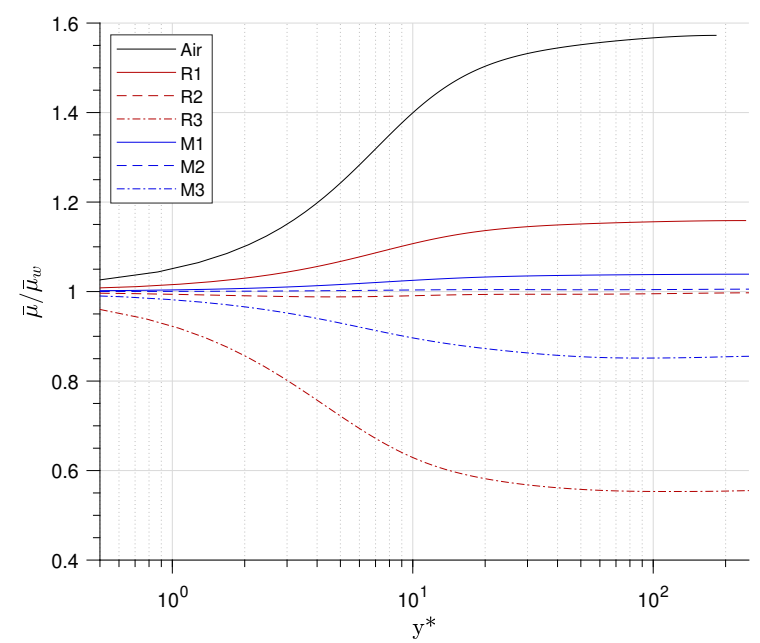

Fig.6 DISTRIBUTION OF AVERAGED DYNAMIC VISCOSITY ALONG THE WALL-NORMAL DIRECTION.

After integrating Eq. 22 along wall distance y, the expression of for pressure ratio shown in Eq. 23 is found. The pressure ratio is associated to $1 / \overline{\hat{p}}_{w}$ and $\int_{0}^{y} \frac{d\left(\bar{\rho} v^{\prime \prime} v^{\prime \prime}\right.}{d y} d y$. If the later part differs slightly between cases, it can be concluded that the larger the wall pressure $\overline{\hat{p}}_{w}$ is the smaller the pressure drop will be.

$$
\begin{gathered}
0=-\frac{d \bar{p}}{d y}+\frac{d}{d y}\left(\bar{\tau}_{2,2}-\bar{\rho} \widetilde{v^{\prime \prime} v^{\prime \prime}}\right) \approx-\frac{d \bar{p}}{d y}-\frac{d\left(\bar{\rho} \widetilde{v^{\prime \prime} v^{\prime \prime}}\right)}{d y} \\
\frac{\bar{p}}{\bar{p}_{w}}=-\frac{1}{\hat{p}_{w}} \int_{0}^{y} \frac{d\left(\bar{\rho} v^{\prime \prime} v^{\prime \prime}\right)}{d y} d y+1
\end{gathered}
$$

The distribution of averaged dynamic viscosity ratio $\bar{\mu} / \bar{\mu}_{w}$ is shown in Fig. 6. It shows that the viscosity increases as $y^{*}$ increases in the Air baseline, R1 and M1, while it decreases in Case R3 and M3. Sciacovelli et al [14] proposed that the former trend is a gas-like behaviour while the latter is a liquid-like behaviour, and that liquid-like behaviours can be observed in dense-gases.

From the result of Fig. 6, it shows that the liquid-like behaviour only exists in the supercritical cases (R3 and M3) of the two organic fluids. By implication there is a transition line from gas-like behaviour to liquid-like behaviour in each organic fluid, which is $\left(\frac{\partial \mu}{\partial T}\right)_{p}=0$ shown in Fig. 7. The slope of the transition line is larger than that of the isobar lines. When fluid works below the transition line, the dynamic viscosity increases with wall distance; otherwise when fluid works above that line, the dynamic viscosity decreases as wall distance is increased.

\section{Reynolds Stress}

The distributions of Reynolds stresses $\widetilde{u^{\prime \prime} u^{\prime \prime}}, \widetilde{v^{\prime \prime} v^{\prime \prime}}, \widetilde{w^{\prime \prime} w^{\prime \prime}}$ and $\widetilde{u^{\prime \prime} v^{\prime \prime}}$ normalized by $u_{\tau}^{* 2}$ are shown in Fig. 8. The normal Reynolds

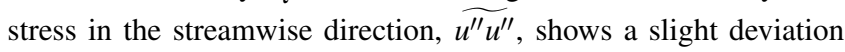
(less than 4\%) between cases (shown in Fig. 8(a)), while the normal Reynolds stress in spanwise and wall-normal directions $\left(v^{\prime \prime} v^{\prime \prime}\right.$ and $\widetilde{w^{\prime \prime} w^{\prime \prime}}$ ) show larger values of deviation (Fig. 8(b) and Fig. 8(c)).

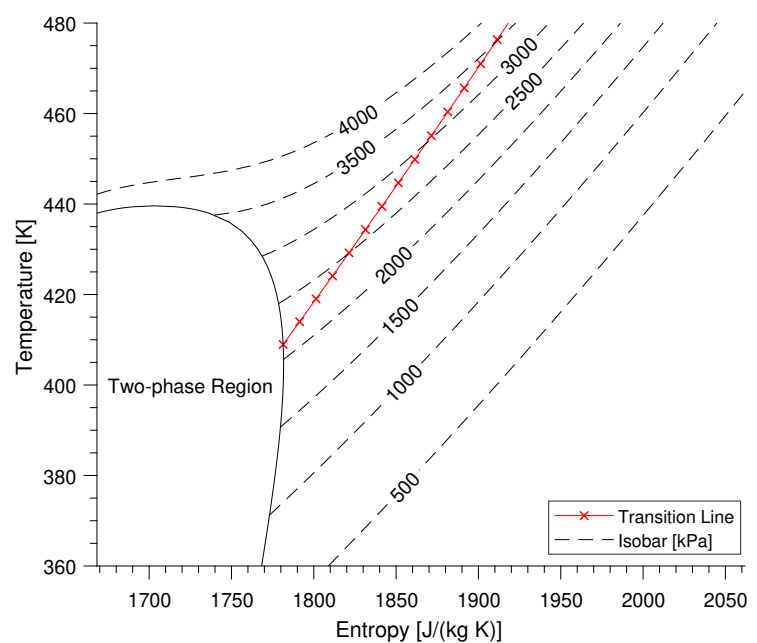

(a) $\mathrm{R} 12337 \mathrm{~d}(\mathrm{E})$

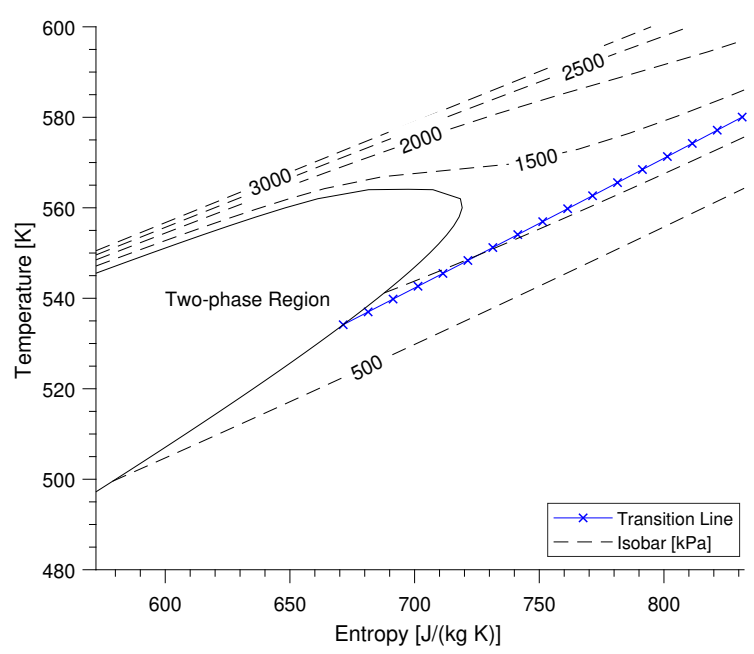

(b) MDM

Fig.7 TRANSITION LINE FOR R1233zd(E) and MDM.

The peak values of $\widetilde{v^{\prime \prime} v^{\prime \prime}}$ in real-gas cases are larger than that in air case by $7-20 \%$. For each fluid, when the real-gas effect increases(from R1 to R3, or M1 to M3), the peak value will increase. Meanwhile, this impact is stronger in R1233zd(E) than in MDM. Real-gas effect also influence the peak values of $\widetilde{w^{\prime \prime} w^{\prime \prime}}$, which are larger by $10-21 \%$ in real-gas cases. The trend of this impact is the same as that of the real-gas effect on $v^{\prime \prime} v^{\prime \prime}$.

The impact of real-gas effect on the normal Reynolds stress in spanwise and wall-normal directions $\left(\widetilde{v^{\prime \prime} v^{\prime \prime}}\right.$ and $\left.\widetilde{w^{\prime \prime} w^{\prime \prime}}\right)$ is associated with the viscosity profile and bulk Reynolds number $R e_{B}$. Patel et al. [13] proposed that if the centre-to-wall viscosity ratio less than 1, the normal Reynolds stress in spanwise and wall-normal directions will increase, and Sciacovelli et al [14] proposed that the increase of bulk Reynolds number $R_{B}$ will lead to a larger peak value of normal Reynolds stress. Both these two effects influence the normal Reynolds stress in the cases of this work, while the viscosity ratio effect seems more predominant in this work, since supercritical case R3 (with the lowest $\bar{\mu}_{c} / \bar{\mu}_{w}$ and lowest $R e_{B}$ ) has the largest maximum $\widetilde{v^{\prime \prime} v^{\prime \prime}}$ and $\widetilde{w^{\prime \prime} w^{\prime \prime}}$ among all cases.

The Reynolds shear stress $\widetilde{u^{\prime \prime} v^{\prime \prime}}$ is larger in two organic fluids by 


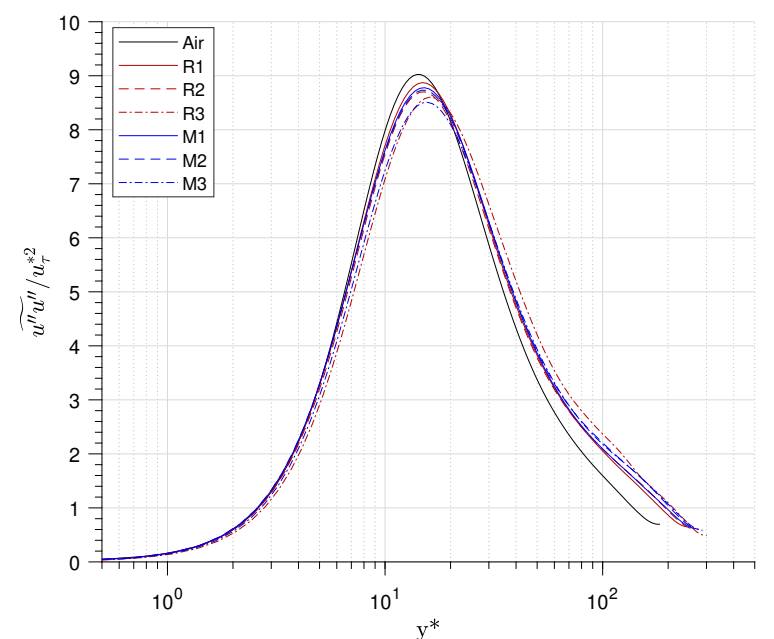

(a)

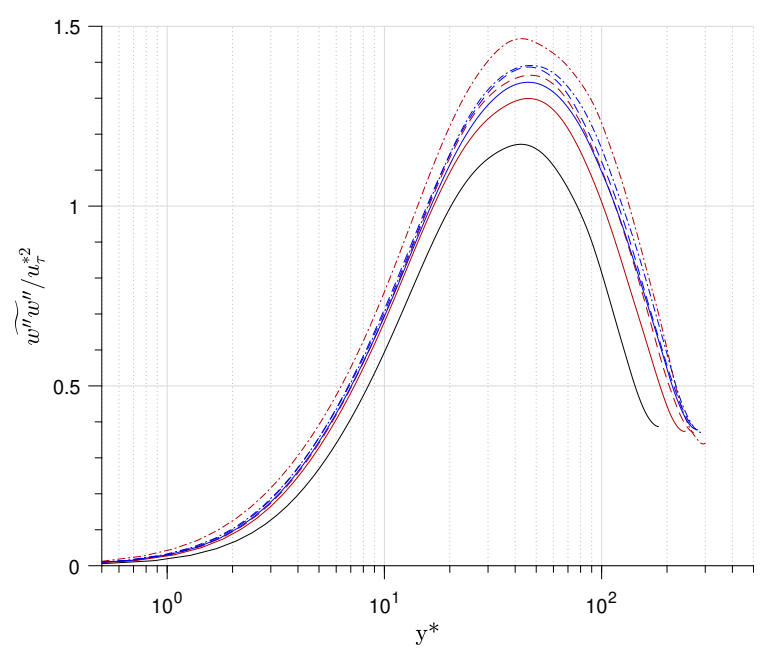

(c)

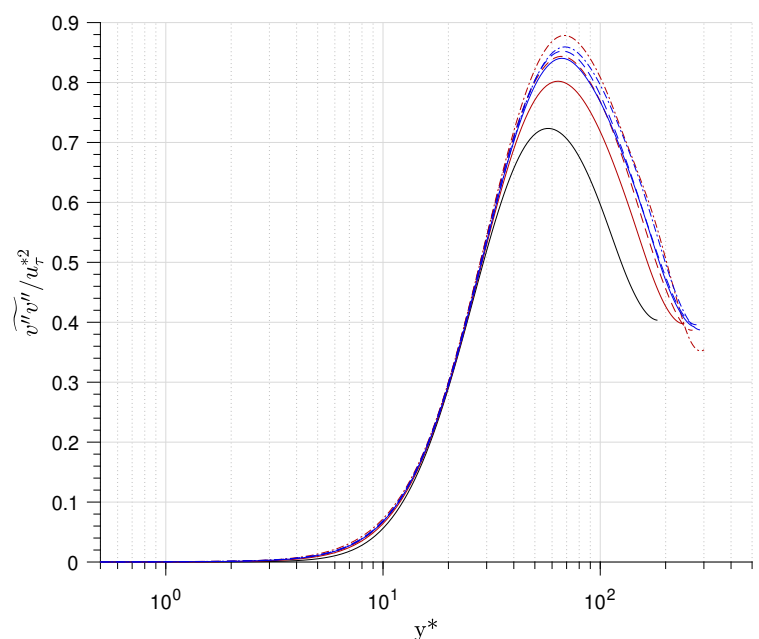

(b)

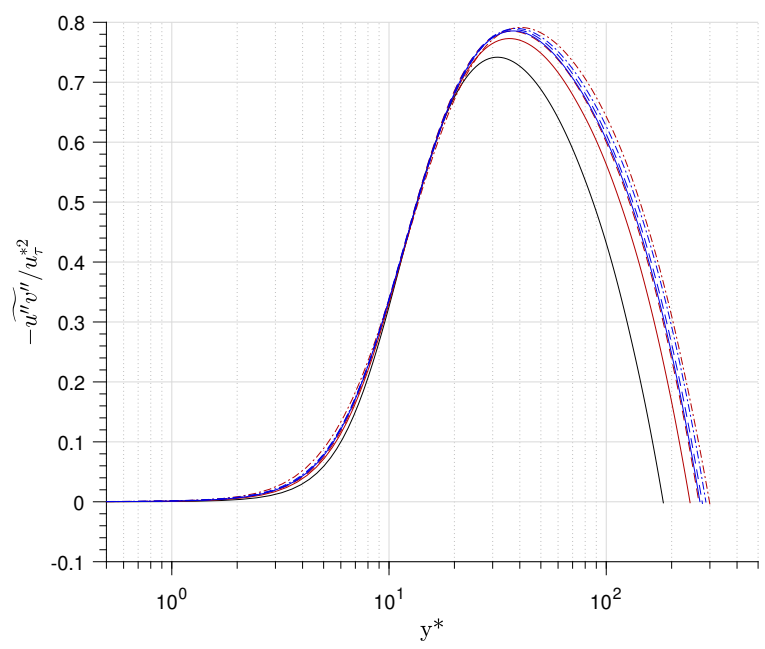

(d)

Fig.8 DISTRIBUTION OF REYNOLDS STRESSES.

(5-8\%) in comparison to air, while the difference between organic fluid cases is not significant. The maximum value of Reynolds shear stress $\widetilde{u^{\prime \prime} v^{\prime \prime}}$ is associated with the viscous shear stress. According to the Reynolds averaged momentum equation in the y direction (Eq. 24), the viscous shear stress and Reynolds shear stress balance the body force together. Since the dynamic viscosity for real-gas cases is smaller in the outer layer, which leads to a smaller viscous shear stress, so the Reynolds shear stress becomes larger compared with air case.

$$
0=\frac{d}{d y}\left(\bar{\tau}_{1,2}-\bar{\rho} \widetilde{u^{\prime \prime} v^{\prime \prime}}\right)+\rho f_{1}
$$

\section{Turbulence Energy}

The Reynolds averaged turbulence energy equation for fully developed channel flow is shown in Eq. 25. The first term is turbulence energy production $P_{k}$; the second term is the summary of turbulent diffusion, velocity-pressure diffusion, and viscous diffusion; the third term is energy dissipation $\varepsilon_{k}$; and the last three terms are compressibility-related terms. The turbulence energy production term $P_{k}$ and dissipation term $\varepsilon_{k}$ are discussed in this work, which are defined in Eq. 26 and Eq. 27.

$$
\begin{aligned}
0=-\overline{\rho u_{1}^{\prime \prime} u_{2}^{\prime \prime}} \frac{\partial \tilde{u_{1}}}{\partial x_{2}} & +\frac{\partial}{\partial x_{2}}\left(-\frac{1}{2} \overline{\rho u_{i}^{\prime \prime} u_{i}^{\prime \prime} u_{2}^{\prime \prime}}-\overline{\rho^{\prime} u_{2}^{\prime \prime}}+\overline{u_{i}^{\prime \prime} \tau_{i, 2}^{\prime}}\right) \\
& -\overline{\tau_{i, 2}^{\prime} \frac{\partial u_{i}^{\prime \prime}}{\partial x_{2}}}-\overline{u_{2}^{\prime \prime}} \frac{\partial \bar{p}}{\partial x_{2}}+\overline{p^{\prime} \frac{\partial u_{i}^{\prime \prime}}{\partial x_{i}}}+\overline{u_{i}^{\prime \prime}} \frac{\partial \overline{\tau_{i, 2}}}{\partial x_{2}}
\end{aligned}
$$

The distribution of turbulence energy production $P_{k}$ is shown in Fig. 9(a). It shows that the fluid property do not significantly influence non-dimensional turbulence energy production, with a maximum $P_{k}$ difference of less than $5 \%$ across all cases. The distribution of turbulence energy dissipation $\varepsilon_{k}$ is shown in Fig. 9(b). The difference between the air and real-gas cases are significant in the viscous sublayer and buffer layer $\left(y^{*}<30\right)$, with the magnitude decreasing in the outer layer $\left(y^{*}>30\right)$. The non-dimensional dissipation at the wall is $5-13 \%$ higher in real-gas 
cases than air. For each organic case, the non-dimensional dissipation in the viscous sublayer and buffer layer increases as the real-gas effect increases (from R1 to R3; M1 to M3).

The turbulence energy production-to-dissipation ratio $-P_{k} / \varepsilon-$ 1 is shown in Fig. 10. A clear peak is demonstrated at $y^{*} \approx 10$ across all cases, followed by a reduction to nearly zero as $y^{*} \approx 50$ is reached. In the region $y^{*}>50$, the air case remains stable before reducing to -1 , while those for real-gas cases will increase slowly before demonstrating the same decrease (albeit at higher values of $y^{*}$ ). The position of the start point for the second drop is related to $R e_{\tau, C}^{*}$, and the larger the $R e_{\tau, C}^{*}$ the later the second drop will be.

$$
\begin{gathered}
P_{k} \equiv-\overline{\rho u_{i}^{\prime \prime} u_{2}^{\prime \prime} \frac{\partial \tilde{u}_{i}}{\partial x_{2}}} \\
\varepsilon \equiv-\overline{\tau_{i, 2}^{\prime} \frac{\partial u_{i}^{\prime \prime}}{\partial x_{2}}}
\end{gathered}
$$

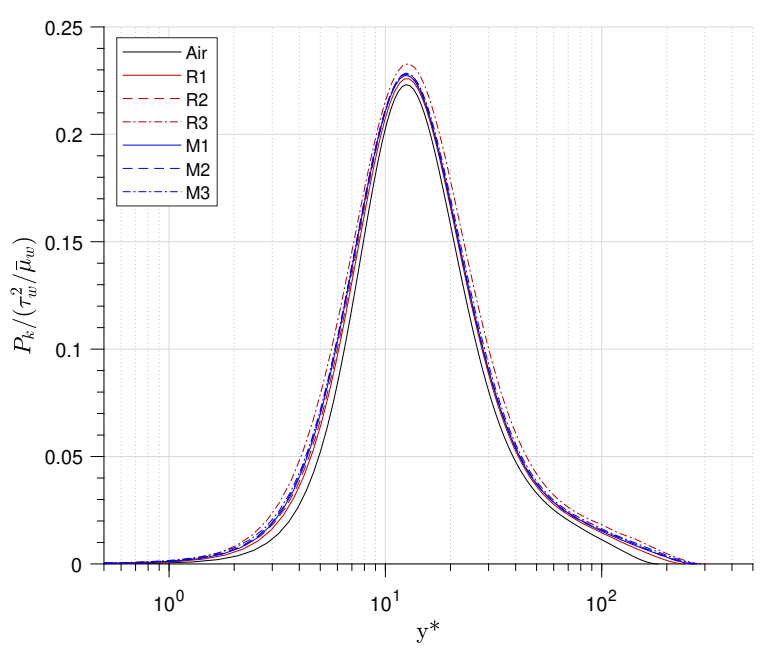

(a)

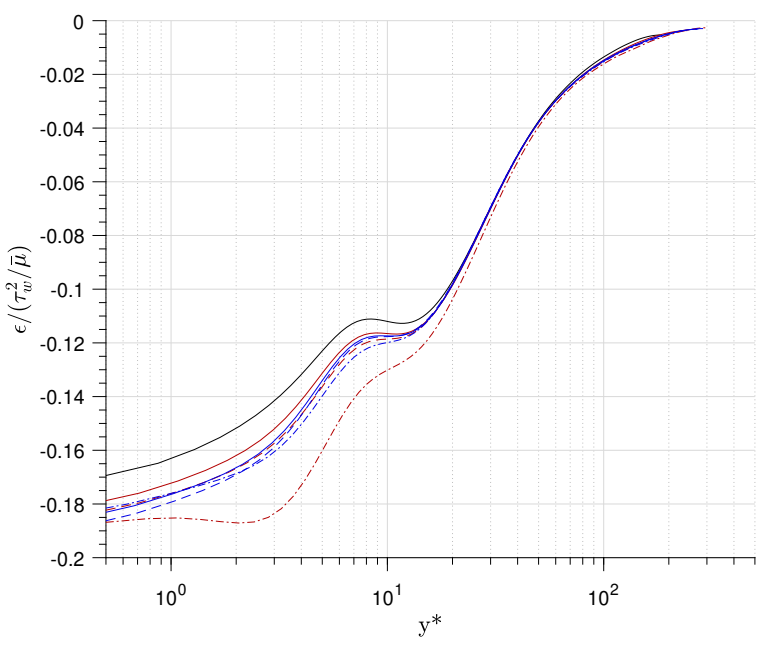

(b)

Fig.9 DISTRIBUTION OF TURBULENCE ENERGY PRODUCTION, (b) DISSIPATION.

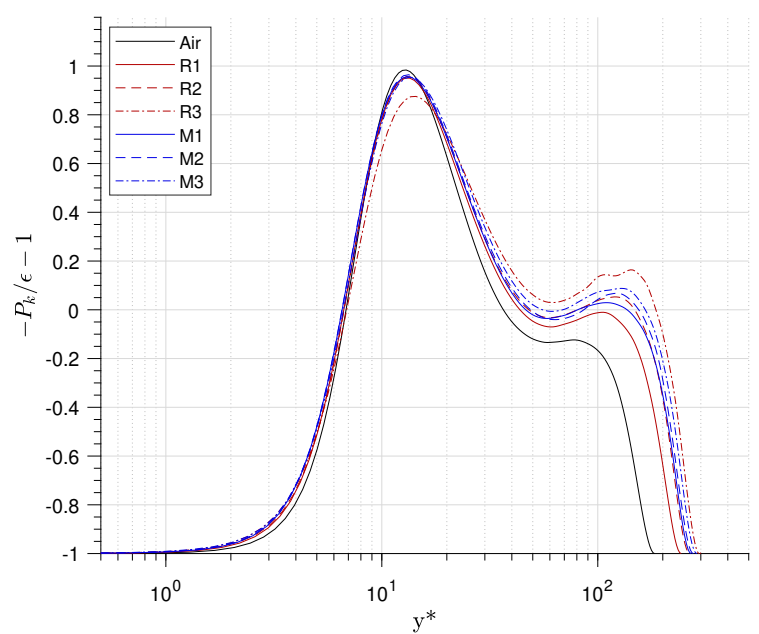

Fig.10 DISTRIBUTION OF TURBULENCE ENERGY PRODUCTION - TO - DISSIPATION RATIO.

\section{Turbulence Viscosity Hypothesis}

The evaluation of the turbulence viscosity hypothesis for the standard two-function RANS model $(k-\varepsilon$ and $k-\omega)$ is a focus of study in this work. The turbulence viscosity $v_{t}$ is defined as the ratio of Reynolds shear stress to the gradient of the averaged velocity, whose simplified expression in the full developed channel flow is shown in Eq. 28. In the standard $k-\varepsilon$ model [22], the turbulence viscosity $v_{t}$ is calculated by the turbulence kinetic energy $k$ and dissipation $\varepsilon$ (Eq. 29), which are solved by equations for $k$ and $\varepsilon$. The constant $C_{\mu}$ is equal to 0.09 .

The ratio $v_{t} \varepsilon / k^{2}$ is calculated for each case and shown in Fig. 11. It shows that in the region $y^{*}<70$, the ratio is lower than 0.09 for all cases, but approximately equal to 0.09 in the region $y^{*}>70$ within deviation bands of $\pm 10 \%$.

With the data for R1233zd(E) and MDM collected so tightly around the constant $C_{\mu}$ line across all cases, there is little evidence to suggest that real-gas effects have a significant impact on this value. A conclusion can therefore be made that the assumption $C_{\mu}=0.09$ is still suitable in the standard two-function RANS model $(k-\varepsilon$ and $k-\omega)$ within the outer layer region at $y^{*}>70$ for the organic fluids within this study.

$$
\begin{gathered}
\widetilde{u^{\prime \prime} v^{\prime \prime}}=v_{t} \frac{d \tilde{u}}{d y} \\
v_{t}=C_{\mu} \frac{k^{2}}{\varepsilon}
\end{gathered}
$$

\section{CONCLUSIONS}

This work studied the fully developed compressible turbulent channel flow of two organic fluids, R1233zd(E) and MDM, by means of Direct Numerical Simulation. Three cases for each fluid are set up at the same global Reynolds number $\left(R e_{G}=4880\right)$ and global Mach number $\left(M a_{G}=2.25\right)$, and they are compared with an air baseline case. Three cases are set up for each organic vapour, representing thermodynamic states far from, close to and inside the supercritical region, and these cases refer to weak, normal and 


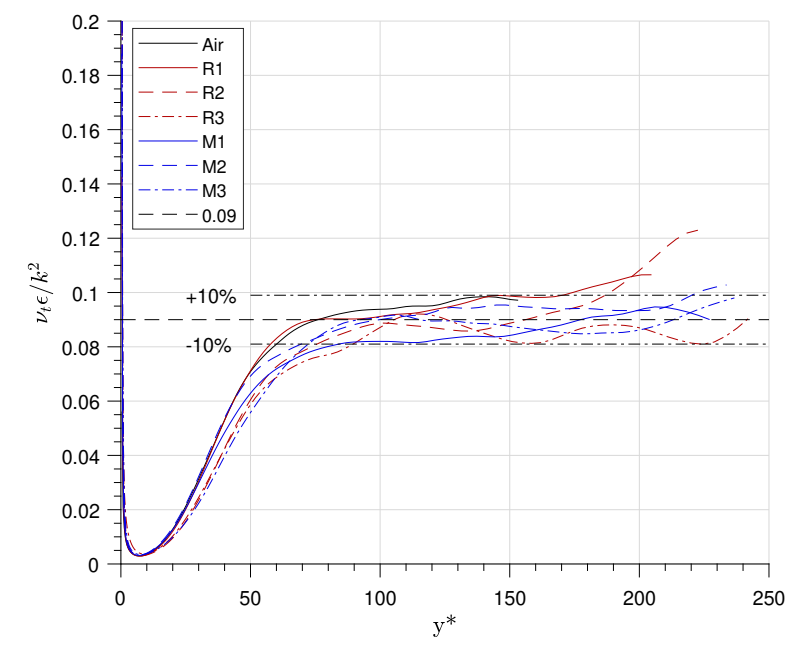

Fig.11 DISTRIBUTION OF $v_{t} \varepsilon_{k} / k_{t}^{2}$ ACROSS ALL CASES.

strong real-gas effect in each fluid.

The turbulent statistic parameters are analyzed and compared.The results show that the real-gas effect influences the averaged thermodynamic property profile. The averaged centre-to-wall temperature ratio is lower in the organic fluid with larger molecular weight, and this ratio will decrease as real-gas effect increases. The averaged wall-to-centre density ratio is also lower in the organic fluid with larger molecular weight, but increases as real-gas effect increases, which is reversed from the impact on averaged centre-to-wall temperature ratio.

The averaged centre-to-wall viscosity ratio is lower than 1 , which is a kind of 'liquid-like behavior', when the real-gas effect is strong enough (in case R1 and M1). There exists a transition line $\left(\frac{\partial u}{\partial T}\right)=0$ for each organic fluid. when working condition is above this line (strong real-gas effect), the viscosity at channel centre is lower than at wall.

The real-gas effect does not influence the normal Reynolds stress in the streamwise direction, but it has an obvious impact on the other two normal Reynolds stress and the Reynolds shear stress. The peak values of the wall-normal Reynolds stress $\widetilde{v^{\prime \prime} v^{\prime \prime}}$ in real-gas cases are larger than that in air case by $7-20 \%$, and the peak values of the spanwise Reynolds stress $\widetilde{w^{\prime \prime} w^{\prime \prime}}$ in real-gas cases are larger by $10-21 \%$. The real-gas effect influences the viscosity profile, therefore, increases $\widetilde{v^{\prime \prime} v^{\prime \prime}}$ and $\widetilde{w^{\prime \prime} w^{\prime \prime}}$. The Reynolds shear stress $\widetilde{u^{\prime \prime} v^{\prime \prime}}$ is also increase by the real-gas effect since its peak value is $5-8 \%$ larger in two real-gas fluids than in air.

The turbulence kinetic energy dissipation $\varepsilon_{k}$ in the viscous sublayer and buffer sublayer $\left(y^{*}<30\right)$ can be impacted by the real-gas effect, which will increase as real-gas effect increases. The turbulence viscosity hypothesis within standard two-function RANS model $(k-\varepsilon$ and $k-\omega)$ is checked in these two organic fluids, and the result shows that it is still suitable in the outer layer $y^{*}>70$ with a constant $C_{\mu}=0.09$ for all real-gas cases, with an error in $\pm 10 \%$.

\section{ACKNOWLEDGEMENT}

The DNS code in this research is developed together with X. Li. The research was funded in part by the China Scholarship Council (CSC).

\section{APPENDIX}

Validation of the Simulation Method

The simulation method is validated by applying it on a compressible turbulent channel flow case of air, of which $R e_{B}=$ 4880 and $M a_{B}=3.0$. The domain size and grid are the same as that used in this work. The result of this case is compared with the DNS data of Coleman [23] and shown in Fig. 12. It shows that the mean velocity, root-mean-square of velocity and Reynolds shear stress distribution follow the result of Coleman, and the difference is within an acceptable range.

\section{$\underline{\text { References }}$}

[1] P.Colonna, S. Rebay, J. Harinck and A. Guardone, 2006, "Real-gas effects in ORC turbine flow simulations: influence of thermodynamic models on flow fields and performance parameters", ECCOMAS CFD 2006 Conference, Egmond aan Zee, NL.

[2] P.Colonna, J. Harinck, S. Rebay and A. Guardone, 2008, "Real-gas effects in organic Rankine cycle turbine nozzles", Journal of Propulsion and Power, Vol. 24, No. 2, pp. 282-294.

[3] A.P. Wheeler and J. Ong, 2014, "A study of the three-dimensional unsteady real-gas flows within a transonic ORC turbine", 2014, ASME Turbo Expo 2014: Turbine Technical Conferemce and Exposition, Dusseldorf, Germany.

[4] G. Persico, M. Pini, V. Dossena, P. Gaetani, 2015, "Aerodynamics of centrifugal turbine cascades", Journal of Engineering for Gas Turbines and Power, Vol. 137, No. 11.

[5] M.S. Cramer and L.M. Best, 1991, "Steady, isentropic flows of dense gases", Physics of Fluids A: Fluid Dynamics, Vol. 3, No. 1, pp. 219-226.

[6] A. Kluwick, 2004, "Internal flows of dense gases", Acta Mechanics, Vol. 169, No. 1-4, pp. 123-143.

[7] P.Colonna and A. Guardone, 2006, "Molecular interpretation of nonclassical gas dynamics of dense vapors under the van der Waals model", Physics of Fluids, Vol. 18, No. 5, 056101.

[8] P.A. Thompson, 1971, "A fundamental derivative in gasdynamics", Physics of Fluids, Vol. 14, No. 9, pp. 1843-1849.

[9] H.A. Bethe, 1942, "Office of Scientific Research and Development"

[10] Y.B. Zeldovich and Y.P. Raizer, 1965, "Physics of shock waves and high-temperature hydrodynamic phenomena", Academic Press, UK.

[11] J. Kim, P. Moin and R. Moser, 1987, "Turbulence statistics in fully developed channel flow at low Reynolds number", Journal of Fluid Mechanics, Vol. 177, pp. 133-166. 


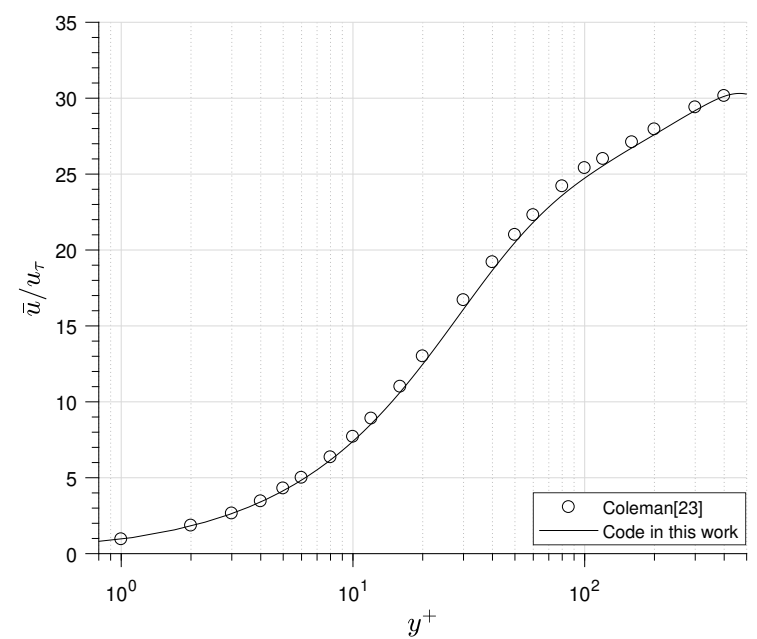

(a)

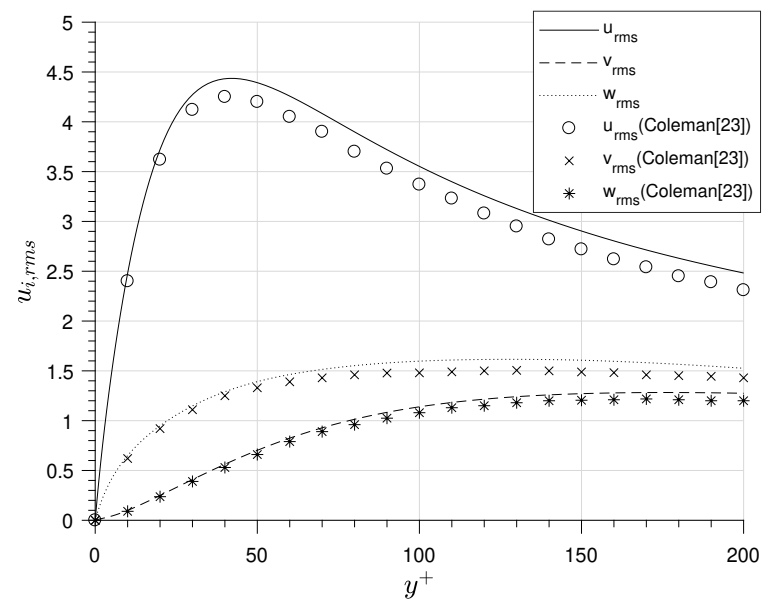

(b)

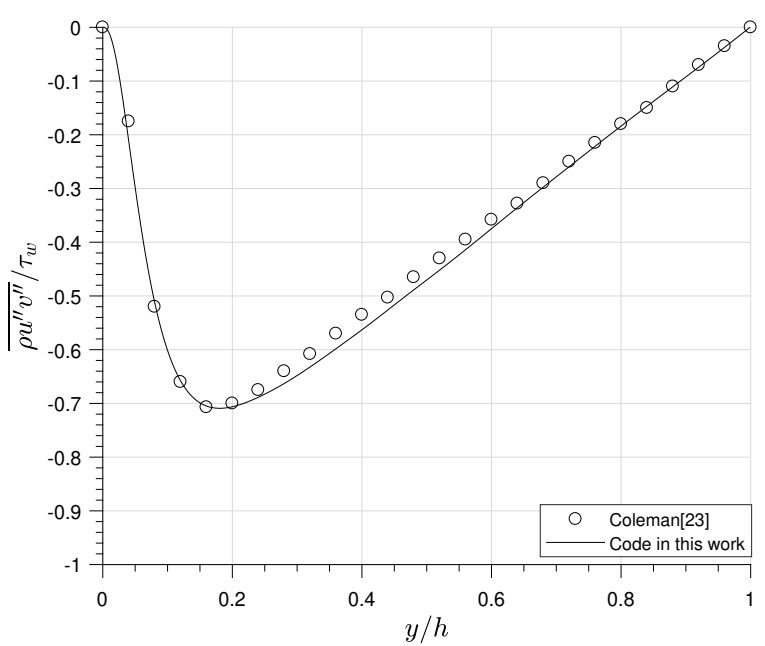

(c)

Fig. 12 VALIDATION OF THE METHOD (a) MEAN VELOCITY, (b) ROOT-MEAN-SQUARE OF VELOCITY AT DIFFERENT DIRECTION, (c) REYNOLDS SHEAR STRESS.

[12] P.G. Huang, G.N. Coleman and P. Bradshaw, 1995, "Compressible turbulent channel flows: DNS results and modelling”, Journal of Fluid Mechanics, Vol. 305, pp. 185-218.
[13] A. Patel, J.W. Peeters, B.J. Boersma and R. Pecnik, 2015, "Semi-local scaling and turbulence modulation in variable property turbulent channel flows", Physics of Fluids, Vol. 27, No. 9, 095101.

[14] L. Sciacovelli, P. Cinnella and X. Gloerfelt, 2017, "Direct numerical simulations of supersonic turbulent channel flows of dense gases", Journal of Fluid Mechanics, Vol. 821, pp. 153-199.

[15] E.W. Lemmon, M.L Huber, M.O. McLinden, 2007 "NIST Standard Reference Database 23: Reference Fluid Thermodynamic and Transport Properties", National Institute of Standards and Technology.

[16] X. Li, Y. Ma and D. Fu, 2001, "DNS and scaling law analysis of compressible turbulent channel flow", Science in China Series a: Mathematics, Vol. 44, No. 5, pp. 645-654.

[17] F. Zonta, C. Marchioli, and A. Soldati ,2012, ”Modulation of turbulence in forced convection by temperature- dependent viscosity", Journal of Fluid Mechanics, vol 697, pp 150-174.

[18] J. Lee, S.Y. Jung, H.J. Sung, and T.A. Zaki, 2013, "Effect of wall heating on turbulent boundary layers with temperature-dependent viscosity", Journal of Fluid Mechanics, vol.726,pp 196-225.

[19] A.S. Monin, and A.M. Yaglom, 1975, "Statistical Fluid Mechanics: Mechanism of Turbulence, book 2", MIT Press.

[20] W. Sutherland, 1893, "The viscosity of gases and molecular force", The London, Edinburgh and Dublin Philosophical Magazine and Journal of Science, Vol. 36, No. 223, pp. 507-531.

[21] E.R. Van Driest, 2003 "Turbulent boundary layer in compressible fluids", Journal of Spacecraft and Rockets, Vol. 40, No. 6, pp. 1012-1028.

[22] B.E. Launder and D.B. Spalding, 1972, "Lectures in Mathematical Models of Turbulence", Academic Press, London, UK.

[23] G.N. Coleman, J. Kim, and R. D. Moser, 1995, ”A numerical study of turbulent supersonic isothermal-wall channel flow", Journal of Fluid Mechanics, Vol 305, pp 159-184. 